\title{
Prevalencia de anticuerpos contra Leptospira en población urbana humana y canina del Departamento del Tolima
}

The prevalence of antibodies against Leptospira in urban human and canine populations from the Tolima Department

\author{
Marlyn H. Romero ${ }^{1}$, Jorge A. Sánchez ${ }^{1}$ y Linda C. Hayek ${ }^{2}$
}

1 Departamento de Salud Animal, Facultad de Ciencias Agropecuarias, Universidad de Caldas. Manizales, Colombia.marlyn.romero@ucaldas.edu.co,jorge.sanchez@ucaldas.edu.co

2 Laboratorio ANIMED Ltda. Bogotá, Colombia. linda.hayek@carval.com.co

Recibido 17 Julio 2009/Enviado para Modificación 20 Febrero 2010/Aceptado 23 Marzo 2010

\section{RESUMEN}

Objetivo Determinar la seroprevalencia de anticuerpos contra Leptospira sp. y los serovares dominantes, en población urbana humana y canina de tres municipios del departamento del Tolima, Colombia.

Materiales y Métodos Se realizó un estudio epidemiológico trasversal con selección de sujetos por conveniencia en 62 barrios. Se obtuvieron muestras de 850 personas.y 850 caninos durante los meses de junio, julio y agosto de 2007, las cuales fueron procesadas utilizando la prueba de microaglutinación (MAT). En la prueba se incluyeron 5 serovares: Hardjo, Pomona, Grippotyphosa, Canicola, Icterohaemorrhagiae y Bratislava. La prueba se interpretó como positiva por la presencia de una aglutinación $\geq 50 \%$ de las leptospiras con uno o más serovares, en una dilución del suero $\geq 100$ para las dos especies.

Resultados Se encontró evidencia de infección en el 6 \% de la población humana y en el $21,4 \%$ de los caninos, con al menos uno de los serovares. La mayor reactividad fue para los serovares Pomona y Grippotyphosa para humanos y caninos. Se presentaron coaglutinaciones en el $13,7 \%$ de los sueros humanos y en el $4,4 \%$ de los caninos. Fue evidente la disminución de la frecuencia de aglutinación de los serovares Canicola e Icterohaemorrhagiae en los perros.

Conclusiones Se observa un cambio en el patrón de presentación de la leptospirosis en poblaciones urbanas, siendo necesario fortalecer la vigilancia epidemiológica activa y pasiva, la implementación de pruebas rutinarias de diagnóstico y medidas de prevención específica en los reservorios animales.

Palabras Clave: Estudios seroepidemiológicos, leptospirosis, factores de riesgo (fuente: DeCS, BIREME).

\section{ABSTRACT}

Objective Determining Leptospira sp. antibody seroprevalence and prevalent serovars in human and canine populations from three municipalities in the Tolima department of Colombia. 
Materials and Methods This was a cross-sectional study (subjects being selected by convenience sampling in 62 districts). Samples were collected from 850 dogs and 850 people during June, July and August 2007; these were processed using a microscopic agglutination test (MAT) and five serovars: Hardjo, Pomona, Grippotyphosa, Canicola, Icterohaemorrhagiae and Bratislava. MAT was considered positive when $50 \%$ or more leptospira were agglutinated with one or more serovars in a 1:100 serum dilution in both species.

Results At least one serovar showed evidence of infection in $6 \%$ of the people and $21.4 \%$ of canines. The most prevalent serovars were Pomona and Grippotyphosa in two populations. Co-agglutinations were present in $49 \%$ of human sera and $19.2 \%$ of canine samples. Decreased Canicola and Icterohaemorrhagiae serovar agglutination frequency was evident in dogs.

Conclusions There was a change in leptospirosis pattern in urban populations. Active and passive surveillance must be strengthened, routine diagnostic tests implemented and preventative measures taken in specific animal reservoirs.

Key Words: Sero-epidemiological study, risk factor, leptospirosis (source: $M e S H, N L M$ ).

$\mathrm{L}$

a leptospirosis es una de las zoonosis más difundidas en el mundo y de gran impacto en salud pública $(1,2)$. Su distribución es mundial, pero se presenta con mayor frecuencia en los trópicos debido a factores ambientales, climáticos y sociales que favorecen su transmisión $(3,4)$. En la actualidad la epidemiología de la enfermedad ha cambiado, especialmente en ambientes ocupacionales y rurales en donde los riesgos asociados han disminuido, pero se ha incrementado el reporte de brotes en zonas urbanas y en poblaciones con diferentes niveles de riesgo, siendo considerada como una de las enfermedades infecciosas reemergentes $(2,5,6)$.

Los reservorios naturales de las Leptospiras son los animales silvestres, especialmente roedores, y los animales domésticos, particularmente bovinos, porcinos y caninos, que pueden actuar como hospedadores de mantenimiento o accidentales, participando en la transmisión de diferentes serovares de Leptospira (5-7). La infección en humanos se presenta por contacto directo de las mucosas con orina de los animales infectados o indirectamente a través de agua o suelos contaminados (7-9). Los caninos se comportan como importantes reservorios de Leptospira sp y fuentes de infección para el hombre, especialmente en población general, por su estrecho contacto con el hombre en ambientes de riesgo no ocupacional $(8,10)$.

La leptospirosis en Colombia no es una enfermedad de notificación obligatoria en el Sistema de Vigilancia en Salud Pública SIVIGILA, sin embargo, se han efectuado estudios de exposición ocupacional que han evidenciado su 
importancia en poblaciones expuestas a riesgo $(11,15)$. Son pocos los trabajos desarrollados en población general asintomática y en reservorios caninos como estrategia de Vigilancia epidemiológica activa en áreas endémicas, información de importancia para determinar la magnitud del problema y las características epidemiológicas de la enfermedad que permitan establecer medidas de control efectivas. Los objetivos del presente estudio fueron: 1) establecer la prevalencia de la infección por Leptospira en población general y canina de los municipios de Piedras, Mariquita y Lérida mediante la técnica de Microaglutinación (MAT) y 2) Identificar los serovares presentes.

\section{MATERIALES Y MÉTODOS}

\section{Área de Estudio}

El trabajo se desarrolló en los municipios de Lérida, Mariquita y Piedras del departamento del Tolima, localizado en las coordenadas $02^{\circ} 52^{\prime} 59^{\prime \prime}$ y $05^{\circ} 19^{\prime} 59^{\prime \prime}$ latitud norte, y los $74^{\circ} 24^{\prime} 18^{\prime \prime}$ y $76^{\circ} 06^{\prime} 23^{\prime \prime}$ longitud oeste. Tienen una temperatura promedio de $26{ }^{\circ} \mathrm{C}$ y una precipitación promedio anual de 1500 a 2000 m.m.

\section{Población humana}

Con la coordinación del personal de la Secretaría Departamental de Salud del Tolima de cada municipio, se trabajó con los líderes comunitarios de 62 barrios para socializar los objetivos del proyecto. Se seleccionó una muestra por conveniencia conformada por 850 personas (350 de Mariquita, 400 de Lérida y 100 de Piedras), con edades comprendidas entre los 15 y 76 años, haciendo un barrido sanitario casa a casa durante los meses junio, julio y agosto de 2007. Sólo una persona de cada vivienda fue muestreada, seleccionada al azar mediante la generación de números aleatorios por calculadora. A cada persona vinculada al estudio se le leyó un protocolo de consentimiento informado el cual estaba aprobado por el comité de bioética de la Secretaria Departamental de Salud de la Gobernación del Tolima, acto seguido se obtuvo su consentimiento para participar en el trabajo y para la toma de las muestras de sangre por parte de funcionarios de los hospitales municipales vinculados.

Población canina

Fueron estudiados 850 caninos de los tres municipios. Se obtuvo el consentimiento informado de los propietarios para realizar la toma de muestras de sangre, siguiendo las normas éticas para el manejo de animales establecidas 
en la Ley 84 de 1989. Se incluyeron en el trabajo los caninos que no habían sido inmunizados contra la leptospirosis.

Prueba de microaglutinación (MAT)

La prueba MAT se realizó de acuerdo a las normas de la Organización Mundial de la Salud, utilizando los serogrupos más comunes en el país: $L$. hardjo, $L$ Pomona, L grippotyphosa, $L$. canicola, $L$. icterohaemorrhagiae y $L$. Bratislava. La prueba se interpretó como positiva por la presencia de una aglutinación $\geq 50 \%$ de las leptospiras con uno o más serovares, en una dilución del suero $\geq 100$ para las dos poblaciones. Entre los sueros positivos que presentaron al mismo tiempo reacciones frente a diferentes serovares, se consideró como causante de la reacción al de mayor título, y como coaglutinaciones a los que presentaron títulos iguales frente a diferentes serovares (10).

Análisis estadístico

Se utilizó el paquete estadístico Epi Info versión 6.04. Se hicieron análisis descriptivos a las variables evaluadas, la comparación entre los grupos se hizo mediante la prueba de ji cuadrado y se estableció un nivel de significancia de $p<0,05$.

\section{RESULTADOS}

La frecuencia de anticuerpos contra Leptospira sp. en la población humana fue del $6 \%$ (51/850) y en los caninos del 21,4\% (182/850). El 37,3\% (n=19) de las personas seropositivas pertenecían al género femenino y el $62,7 \%$ restante al masculino, con edades comprendidas entre los 16 y 70 años; no se presentaron diferencias significativas entre la seroreactividad entre edades, ni género $(p=0.23)$. El municipio con mayor proporción de casos fue Lérida $(62,7 \%)$, seguido de Mariquita (31,3\%) y Piedras (5,9 \%). El serovar más frecuentemente aglutinado por los sueros humanos evaluados fue Pomona (43,1 \%), seguido por Grippotyphosa (17,6\%), sin embargo, el primero fue aglutinado por la totalidad de los sueros humanos positivos (Tabla 1). Así mismo, se presentaron coaglutinaciones en el 13,7\% de los sueros. En los caninos el serovar Grippotyphosa fue el más frecuente $(82,4 \%, 150 / 182)$, seguido de Pomona $(8,2 \%, 15 / 182)$, presentándose coaglutinaciones en el 4,4 \% de sueros positivos. Se observó una baja reactividad a los serovares Canicola $(0,5 \%, 1 /$ 182) e Icterohaemorrhagiae $(2,2 \%, 4 / 182)$. 
Tabla 1. Distribución de los sueros humanos positivos a leptospirosis con la prueba de microaglutinación (MAT) detallando los serovares reaccionantes

\begin{tabular}{|c|c|c|c|c|c|c|c|c|}
\hline & $1 / 100$ & $1 / 200$ & $1 / 400$ & $1 / 800$ & $1 / 1600$ & $>1 / 1600$ & Total & $\%$ \\
\hline $\mathrm{H}$ & & 4 & & & & & 4 & 7,8 \\
\hline$P$ & 3 & 7 & 5 & 1 & 2 & 4 & 22 & 43,1 \\
\hline G & 6 & 3 & & & & & 9 & 17,6 \\
\hline B & 1 & 2 & & & 1 & & 4 & 7,8 \\
\hline $\mathrm{C}$ & & & & & & & 0 & 0,0 \\
\hline I & 3 & & & & & 1 & 4 & 7,8 \\
\hline \multicolumn{9}{|c|}{ Coaglutinaciones } \\
\hline $\mathrm{Pi}$ & & 1 & & & & 1 & 2 & 3,9 \\
\hline $\mathrm{Pg}$ & 2 & 2 & & & & & 4 & 7,8 \\
\hline $\mathrm{Hpi}$ & & & 1 & & & & 1 & 2,0 \\
\hline Pbci & & & 1 & & & & 1 & 2,0 \\
\hline Total & 15 & 19 & 7 & 1 & 3 & 6 & 51 & 100 \\
\hline$\%$ & 29,4 & 37,3 & 13,7 & 2,0 & 5,9 & 11,8 & & \\
\hline
\end{tabular}

Hardjo h, Pomona p, Grippotyphosa g, Bratislava b, Caninola c, Icterohaemorrhagiae i.

\section{DISCUSIÓN}

La epidemiología de la leptospirosis ha sufrido cambios significativos en recientes años y en la actualidad ha emergido como una zoonosis de importancia global, que se presenta en ambientes urbanos $(5,16)$, aspecto que se evidencia en los municipios del departamento del Tolima evaluados, en donde se estableció una seroprevalencia del $6 \%$ en población general urbana. La prevalencia de leptospirosis observada en la población fue más baja que la reportada en habitantes de barrios periféricos de Cali (23,3\%) (2) y en el Urabá Antioqueño $(12,5 \%)(17)$, similar a la establecida en pacientes urbanos de los departamentos del Quindío por MAT (18) y Atlántico (19), pero comparable con reportes recientes de otros países latinoamericanos en áreas urbanas $(20,21)$.

La prevalencia de los diferentes serovares de Leptospira en la población humana depende de los reservorios animales presentes y de los serovares que éstos portan, así como de las condiciones ambientales locales, la ocupación, el grado de contacto entre los hospedadores de mantenimiento y accidentales, y las prácticas sanitarias de la población a riesgo $(5,16,22)$. Los serovares más prevalentes en la población humana evaluada en el presente estudio fueron Pomona y Grippotyphosa (Tabla 1), asociados con los reservorios cerdos y bovinos $(16,20)$, la primera; y a bovinos, mapaches y marsupiales la segunda $(16,18)$, sin embargo, es muy baja la reactivad al serovar Icterohaemorrhagiae $(5,9 \%)$, el cual tradicionalmente ha estado vinculado con la infección humana y su relación con los roedores como los principales reservorios $(5,16,19)$, este último serovar en el país ha sido prevalente en estudios longitudinales realizados 
en el departamento del Atlántico (19), siendo asociado con el incremento de la severidad de la enfermedad $(1,6)$. La alta reactividad ante el serovar Grippotyphosa ha sido también establecida en otras regiones del país $(2,17)$.

Es importante resaltar que el serovar Pomona fue aglutinado por el total de las muestras humanas seropositivas (Tabla 1) y que éste ha sido aislado en Europa de reservorios silvestres y de los roedores sinantrópicos Rattus rattus y $R$. norvergicus (6); en Argentina fue el segundo serovar de importancia detectados en un estudio epidemiológico efectuado durante siete años de vigilancia epidemiológica de la enfermedad (20), vinculándose además con los casos de leptospirosis severa que producen falla renal (22). No debemos olvidar que el conocimiento de los serovares prevalentes y sus hospedadores de mantenimiento es esencial para el entendimiento de la epidemiología de la leptospirosis y en la implementación de las medidas de control de la enfermedad en una región $(5,16)$.

Se ha discutido sobre la emergencia de la leptospirosis canina en Norte América y Canadá asociada con los serovares Pomona y Grippotyphosa $(5,10,23)$, presumiblemente adquirida de mofetas, mapaches y zorrillos $(5,23)$; así como el descenso de los casos asociados con los serovares Icterohaemorrhagiae y Canicola (23). Esta información es concordante con los resultados obtenidos en el presente estudio, en donde se evidencia una baja dominancia de los serovares Icterohaemorrhagiae y Canicola, que son los más frecuentemente reportados en los estudios epidemiológicos efectuados en poblaciones caninas en Colombia (9) y en países latinoamericanos como Argentina (24), 1997; México (8), Chile (10) y Perú (21). Se sugiere que la disminución de los casos asociados con estos dos serovares está relacionada con la vacunación continuada de las poblaciones caninas con la vacuna bivalente que los incluye, que induce inmunidad específica para éstos, pero que puede favorecer la presencia potencial de otros serovares en las poblaciones caninas susceptibles $(23,25)$, aspecto que se puede estar presentando en la región del Tolima incluida en el estudio. Con relación a los serovares Pomona y Grippotyphosa, han estado vinculados con reservorios silvestres como ya se indicó anteriormente. En Norte América, Canadá y la Unión Soviética se ha sugerido que la alta prevalencia de estos serovares en la población canina es debida al contacto previo de los perros con los reservorios silvestres, así como en el caso del serovar Pomona, por el contacto indirecto con fuentes de agua contaminadas con excretas porcinas (23). 
Se presentaron coaglutinaciones en el 13,7 \% (Tabla 1) de los sueros humanos y en menor proporción en los sueros caninos $(4,4 \%)$, las cuales indican reacciones cruzadas por la presencia de antígenos de membrana comunes entre los diferentes serovares y serogrupos $(5,9,25)$, o por anticuerpos que persisten de contactos infecciosos previos con diferentes leptospiras, que pueden generar reacciones paradójicas que hacen difícil la interpretación del MAT (2), siendo necesario el aislamiento de las leptospiras en cultivo y la tipificación para establecer los serovares circulantes en el Departamento del Tolima. Así mismo, teniendo en cuenta que la población humana mostró similares perfiles de positividad para Leptospira a los encontrados en caninos, sería conveniente desarrollar futuros estudios para establecer si los perros podrían actuar como intermediarios entre los reservorios naturales (marsupiales silvestres) y el hombre, como lo han sugerido otros investigadores en Perú (21), así como la necesidad de identificar los reservorios naturales que pueden estar participando en el ciclo de transmisión en la zona evaluada.

Se sugieren como medidas de prevención y control de la enfermedad en la población incluida en el estudio, el desarrollo de actividades de promoción dirigidas a población general y a profesionales de la salud, control integrado de roedores y control de la sobrepoblación canina y vacunación de las especies susceptibles. Así como fortalecer el sistema de vigilancia epidemiológica activa y pasiva y el soporte de diagnóstico para reducir el impacto de la leptospirosis

Agradecimientos: Los autores quieren expresar su agradecimiento a la Gobernación del Tolima-Secretaría Departamental de Salud por el financiamiento de esta investigación.

\section{REFERENCIAS}

1. Berlioz-Arthaud A, Kiedrzynski T, Singh N, Yvon J-F, Roualen G, Coudert C, Uluiviti V. Multicentre survery of incidence and public health impact of leptospirosis in the Western Pacific. Trans R Society of Trop Med Hyg. 2007; 101:714-721.

2. Ferro BU, Rodríguez AL, Pérez M, Travi BL. Seroprevalencia de infección por Leptospira en habitantes de barrios periféricos de Cali. Biomédica 2006; 26:250-257.

3. Baranton G, Postic D. Trends in leptospirosis epidemiology in France. Sixty-six years of passive serological surveillance from 1920 to 2003. Int J Infect Dis. 2006; 10:162-170.

4. Pappas G, Papadimitriou P, Siozopoulou V, Christou L, Akritidis N. The globalization of leptospirosis: worldwide incidence trends. Int J Infect Dis. 2008; 12:351-357.

5. Levett PN. Leptospirosis: A forgotten zoonosis? Clin Microbiol Rev. 2004; 4:435-448.

6. Vieira MS, Gama-Simões MJ, Collares-Pereira S. Human leptospirosis in Portugal: a retrospective study of eithteen years. Int J Infect Dis. 2006; 10:378-386. 
7. Abgueguen P, Delbos V, Blanvillain J, Chennebault JM, Cottin J, Fanello S, et al. Clinical aspects and prognostic factors of leptospirosis in adults. Retrospective study in France. J Infect. 2008; 57:171-178.

8. Jiménez-Coello M, Vado-Solis I, Cárdenas-Marrufo MF, Rodríguez-Buenfil JC, Ortega-Pacheco A. Serological Survey of canine leptospirosis in the tropics of Yucatan Mexico using two different tests. Acta Trop. 2008; 106:22-26.

9. Rodríguez AL, Ferro BE, Varona MX, Santafé M. Evidencia de exposición a Leptospira en perros callejeros de Cali. Biomédica 2004; 24:291-295.

10. Silva RF, Riedemann S. Seroprevalencia de leptospirosis canina en perros atendidos en clínicas veterinarias, mediante aglutinación microscópica y comparación con las técnicas de aislamiento e inmunofluorescencia indirecta. Arch. Med. Vet. 2007; 39:269-274.

11. Romero MH, Sánchez JA, Hayek LC. Leptospirosis, brucelosis y toxoplasmosis: Zoonosis de importancia en población ocupacionalmente expuesta. Biosalud 2008; 7:21-27.

12. Nájera S, Alvis N, Babilonia D, Alvarez L, Máttar S. Leptospirosis ocupacional en una región del Caribe colombiano. Salud Publica Méx. 2005; 47(3):240-244.

13. Díaz L, Zapata I, Góngora A, Parra J, Aponte G, Gómez L, et al. Detección de anticuerpos IgM a Leptospira en humanos en riesgo ocupacional en Villavicencio, Meta. Rev. MVZ Córdoba 2008; 13 (1):1120-1127.

14. Góngora A, Parra JL, aponte LH, Gómez LA. Seroprevalencia de Leptospira sp. en grupos de población de Villavicencio, Colombia. Rev. Salud publica 2008; 10(2):269-278.

15. Orrego A, Giraldo de León G, Ríos B, Valencia PA. Leptospirosis en personas de riesgo de quince explotaciones porcinas y de la central de sacrificio de Manizales, Colombia. Arch. Med. Vet. 2003; 35(2):205-213.

16. Bharti AR, Nally J, Ricaldi JN, Mathias M, Díaz MM, Lovett MA, et al. Leptospirosis: a zoonotic disease of global importance. Lancet Infect Dis. 2003; 3:757-769.

17. Agudelo-Flórez P, Restrepo-Jaramillo BN, Arboleda-Naranjo M. Situación de la leptospirosis en el Urabá antioqueño colombiano: estudio seroepidemiológico y factores de riesgo en población general urbana. Cad Saúde Pública 2007; 23(9):2094-2102.

18. Sánchez G, Gómez-Marín JE, Quintero L, Castaño MC. Características clínicas y epidemiológicas de la leptospirosis en el departamento de Quindío, 2005-2006. Infectio 2008; 12(2):325-331.

19. Macías JC, Vergara C, Romero C, Falconar AKI. Comportamiento de la leptospirosis en el departamento de Atlántico (Colombia). Enero de 1999 a marzo del 2004. Salud Uninorte 2005; 20:18-29.

20. Vanasco NB, Schmeling MF, Lottersberger J, Costa F, Ko AI, Tarabla HD. Clinical characteristics and risk factors of human leptospirosis in Argentina (1999-2005). Acta Trop. 2008; 107:255-258.

21. Céspedes M, Chun M, Cano E, Huaranca I, Atoche H, Ortiz H, et al. Prevalencia de anticuerpos contra Leptospira en personas asintomáticas y en perros de Chancay, Lima 2001. Rev Peru Med Exp Salud Publica 2007; 24(4):343-349.

22. Adler B, de la Peña M. Leptospira and leptospirosis. Vet Microbiol. 2010; 140:287-296.

23. Ward MP. Clustering of reported cases of leptospirosis among dogs in the United States and Canada. Prevent Vet Med 2002; 56:215-226.

24. Rubel D, Seijo A, Cernigoi B, Viale A, Wisnivesky-Colli C. Leptospira interrogans en una población canina del Gran Buenos Aires: variables asociadas con la seropositividad. Rev Panam Salud Publica 1997; 2(2):102-106.

25. André-Fontaine G. Canine leptospirosis - Do we have a problem? Vet Microbiol 2006; 117:19-24. 